\title{
Expressões de gênero e o cuidado em saúde mental: perspectiva de profissionais que atuam em Centros de Atenção Psicossocial
}

Gender expressions and mental health care: perspective of professionals who work in Psychosocial Care Centers

Expresiones de género y cuidado de salud mental: perspectiva de profesionales que trabajan en los Centros de Atención Psicosocial

Recebido: 20/09/2021 | Revisado: 01/10/2021 | Aceito: 05/10/2021 | Publicado: 09/10/2021

Suelen de Oliveira Maas
ORCID: https://orcid.org/0000-0002-5759-6672
Faculdades Pequeno Príncipe, Brasil
E-mail:suelenomaas@gmail.com
Luciana Elisabete Savaris
ORCID: https://orcid.org/0000-0002-7408-1187
Faculdades Pequeno Príncipe, Brasil
Universidade Federal do Paraná, Brasil
E-mail:profelusavaris@ gmail.com

\section{Resumo}

As sociedades heteronormativas e patriarcais podem gerar riscos à Saúde Mental da população. O objetivo deste estudo foi o de compreender se diferentes expressões de gênero são consideradas relevantes no acolhimento e escolhas de ofertas terapêuticas em Centros de Atenção Psicossocial. A pesquisa é de natureza qualitativa, exploratório-descritiva e transversal. Os dados foram coletados a partir de Projetos Institucionais e da aplicação de questionário semiestruturado junto a profissionais dos Centros de Atenção Psicossocial numa capital do sul do Brasil, sendo categorizados conforme a Análise de Conteúdo postulada por Bardin. Os resultados apontam que diferentes performances de gênero não são tomadas a priori como aspecto pertinente no acolhimento e na escolha de ofertas terapêuticas. Tal incorporação ocorre somente quando esta demanda se apresenta de forma explícita por usuário/a e mais, quando o/a usuário/a chega ao acolhimento neste dispositivo. Embora profissionais identifiquem a vulnerabilidade relacionada a vivência de múltiplas performances de gênero, não foi possível identificar estratégias institucionais voltadas ao tema.

Palavras-chave: Diversidade de gênero; Centro de atenção psicossocial; Saúde mental.

\begin{abstract}
Heteronormative and patriarchal societies can generate risks to Mental Health. The objective of is understanding whether different gender expressions are considered relevant in the reception and choices of therapeutic offers in "Centros de Atenção Psicossocial" (CAPS). The research is a qualitative, exploratory-descriptive and transversal research. Data were collected from Institutional Projects and from the application of a semi-structured questionnaire with professionals from the aforementioned CAPS, being categorized according to the Content Analysis postulated by Bardin. The results indicate that different expressions of gender are not taken a priori as a relevant aspect, in the reception and choice of therapeutic offers and that its incorporation occurs only when this demand is explicitly presented by the user, and moreover, when this user arrives at the reception on this device. Although professionals identify the vulnerability related to the experiences of different gender expressions, it was not possible to identify institutional strategies focused on the theme.
\end{abstract}

Keywords: Gender diversity; Mental health Services; Mental health.

\section{Resumen}

Las sociedades heteronormativas y patriarcales pueden generar riesgos para la enfermedad mental. El objetivo es comprender si las diferentes expresiones de género se consideran relevantes en la recepción y elección de opciones terapéuticas en "Centros de Atenção Psicossocial" (CAPS). Se trata de una investigación cualitativa, exploratoriadescriptiva y transversal. Se recolectaron datos de Proyectos Institucionales y la aplicación de un cuestionario semiestructurado con profisionales de los CAPS antes mencionados, siendo categorizado segúnpor el Análisis de Contenido portulado por Bardin. los Resultados indican que las diferentes expresiones de género no se toman $a$ priori como un aspecto relevante, en la recepción y elección de ofertas terapéuticas y que su incorporación se produce solo cuando esta demanda es presentada explícitamente por el usuario, y además, cuando este usuario llega a la recepción en este dispositivo. Si bien los profesionales identifican la vulnerabilidad relacionada con las 
experiencias de diferentes expresiones de género, no fue posible identificar estrategias institucionales enfocadas en el tema.

Palabras clave: Diversidad de género; Servicios de salud mental; Salud mental.

\section{Introdução}

O movimento da Reforma Psiquiátrica Brasileira objetivou a garantia de acolhimento, acompanhamento e tratamento humanizado a todos os sujeitos, independentemente de característica, como classe, raça, gênero ou condição física. Preconizou a dissolução gradativa hospitais psiquiátricos, substituindo os leitos por uma rede de atenção psicossocial territorial, longitudinal e transversal (Almeida, 2019; Yasui \& Barzaghi, 2018). Diferentes equipamentos de saúde passam a atender às demandas de Saúde, tais como: Unidades Básicas de Saúde (UBS), Centros de Atenção Psicossocial (CAPS), Unidades de Pronto Atendimento (UPA), Hospitais Gerais, entre outros (Almeida, 2019; Yasui \& Barzaghi, 2018; Brasil, 2011; Amarante, 2015; Maas \& Savaris, 2020).

O CAPS se destaca como equipamento prioritário de cuidado à Saúde Mental, é responsável por um determinado território e desenvolve ações conjuntas de prevenção, promoção e assistência à saúde com as equipes das UBS. O modelo de atenção e cuidado psicossocial, que contrapõe o modelo hospitalocêntrico, amplia os horizontes ao integrar a comunidade e respeitar as singularidades, na construção dos projetos terapêuticos, tendo como focos prioritários a proteção de direitos e a busca pela reinserir socialmente do usuário (Yasui \& Barzaghi, 2018; Andrade, 2014; Amarante \& Nunes, 2018).

Nesta perspectiva de cuidado as questões relacionadas ao social ganham destaque, e o adoecimento psíquico passa a ser compreendido para além da dimensão biológica, em sua "expressão simbólica e construtiva" (Zanello, 2014, p. 44). Valores, crenças e normas sociais são apreendidos como fatores relevantes para compreensão do indivíduo, assim como, seu contexto e seus grupos sociais, com suas expectativas e atitudes que se convertem em preditores de melhor ou pior Saúde Mental. Deste modo, as expressões de gênero podem ser entendidas como elemento imbricado no processo de subjetivação e integram a saúde mental dos indivíduos (Santos, 2009; Nascimento \& Zanello, 2014).

O termo gênero "foi utilizado pela primeira vez no ano de 1955 por John Money para descrever o conjunto de condutas atribuídas aos homens e às mulheres" (Lima, 2011, p. 168). Gênero é um conceito produzido culturalmente que designa aquilo que incorpora o universo feminino e masculino, mas, além disso, também determina o lugar que sujeitos ocupam na sociedade.

Diversas pesquisadoras contemporâneas discutem a relação gênero, sociedade e saúde, dentre estas, destaca-se Judith Butler (2018) que define expressão ou performatividade de gênero como a definição feita social e culturalmente e que delimita como o sujeito se expressa diante do mundo, definição adotada neste estudo. Butler (2018) também problematiza que a performance de gênero aceita socialmente no século XXI ainda é demarcada por uma heterossexualidade compulsória, ou seja, masculino e feminino aceitos como um binarismo, presos ao sexo biológico (pênis/vagina) e com representações sociais associadas a esta norma (Maas \& Savaris, 2020).

Exemplo clássico desse binarismo é oferecido cotidianamente, pois que usualmente "espera-se que meninos gostem de azul, brinquem com carrinhos, e que meninas gostem de rosa e brinquem com bonecas. Espera-se que mulheres sejam sensíveis e usem cabelos compridos, e que homens sejam fortes e não chorem" (Secretaria de Justiça e da Defesa da Cidadania, 2014, p. 13). O gênero aceito socialmente é aquele que mantêm coerência e continuidade entre, "gênero, sexo, prática sexual e desejo", ignorando a diversidade de expressões possíveis (Butler, 2018, p. 43).

Neste estudo, a Teoria das Representações Sociais, formulada por Serge Moscovici embasa a compreensão de gênero adotada pelo senso comum, ao considerar que sujeitos se influenciam mutuamente (Moscovici, [1961] 2011) e que a partir destas influências, se define o entendimento prévio sobre certo objeto, conceito ou pessoa. Cabe ressaltar que profissionais de saúde, também estão sujeitos a estas influências, por estarem inseridos em uma determinada sociedade, cultura e momento 
histórico, o que afeta sua visão de mundo, bem como, seu modo de acolher e intervir nas demandas de saúde (Zanello \& Silva, 2012; Moscovici, 2019; Maas \& Savaris, 2020).

Ao relacionar expressões de gênero ao adoecimento psíquico, diferenças são constatadas (Andrade, Viana \& Silveira, 2006; Rabasquinho \& Pereira, 2007; Santos, 2009; Zanello, 2014). Mulheres apresentam uma prevalência maior de transtornos de humor, enquanto em homens o que se destaca é o abuso/dependência de substâncias psicoativas, transtornos de personalidade, transtorno do controle de impulsos e déficit de atenção. Quando associado à população LGBTQIA+ a vivência de estigmas, preconceitos e discriminação ganham destaque como fatores de risco ao adoecimento psíquico, em especial a quadros de ansiedade, depressão e comportamento suicida (Zanello \& Silva, 2012).

Ao ocupar-se do tema expressões de gênero e Saúde Mental, considerando o modelo de atenção psicossocial de atenção à saúde, este estudo teve como objetivo, compreender se as diferentes expressões de gênero são tomadas como aspecto relevante, no acolhimento e na escolha de ofertas terapêuticas, por profissionais de saúde que atuam em CAPS.

\section{Metodologia}

Trata-se de uma pesquisa qualitativa, de natureza descritiva exploratória, que explica os fenômenos considerando os sistemas sociais e culturais envolvidos (Gil, 2017; Marconi \& Lakatos, 2021) O estudo teve um recorte transversal e ocorreu entre os meses de julho a setembro de 2020.

A coleta de dados foi realizada em três CAPS de uma capital do sul do país. Estes dispositivos estão direcionados ao atendimento de pessoas adultas com transtornos mentais graves e/ou crônicos com ou sem uso de substâncias psicoativas. Para seleção dos CAPS foi utilizado como critério o Índice de Vulnerabilidade das Áreas de Abrangência das Unidades Municipais de Saúde (IVAB) (Decreto n. 638, 2018), sendo selecionado um território de cada categoria do IVAB: alto, médio e baixo, o que permite contemplar territórios da cidade, com características distintas. Participaram do estudo, representantes de diferentes categorias de nível superior que integram as equipes multiprofissionais, sendo profissionais: da Medicina, da Enfermagem, da Psicologia, da Terapia Ocupacional, e do Serviço Social.

Quanto às fontes de dados, a pesquisa dividiu-se em duas etapas: na primeira etapa foram coletados dados secundários, ou seja, fonte documental. Procedeu-se a leitura sistemática dos Projetos Institucionais (PI) de cada CAPS selecionado, buscou-se pelas palavras-chave: gênero(s), masculino, feminino, homem, mulher, transgênero, expressão de gênero, transexual, homossexual, lésbica, gay, bissexuais, acolhimento e ofertas terapêuticas.

$\mathrm{Na}$ segunda etapa foram coletados dados primários a partir da aplicação de dois questionários, sendo um sociodemográfico: data de nascimento; escolaridade; profissão; cargo; tempo que trabalha em CAPS e gênero com o qual se identifica. E o outro questionário, semiestruturado, continha perguntas abertas: o que você compreende por expressão de gênero; o serviço que você trabalha recebe usuários/as com diferentes expressões de gênero? Caso sua resposta seja afirmativa como ocorre o acolhimento, o atendimento e a escolha de ofertas terapêuticas as diferentes expressões de gênero, exemplifique; essa temática já foi problematizada em reunião de equipe ou em discussão de casos? Caso sua resposta seja afirmativa exemplifique. Em sua opinião, questões de gênero se relacionam com o adoecimento psíquico? O que você pensa sobre isso? Na sua prática, já vivenciou essa situação com algum/a usuário/a atendido? Na sua prática em de CAPS, o serviço é mais buscado por homens ou mulheres, cis ou trans? Existe diferença quanto à adesão? Gostaria de deixar alguma contribuição que não foi abordada durante o questionário?

Os dados coletados foram trabalhados a partir da Análise de Conteúdo conforme proposta por Bardin, técnica que permite compreender modelos, características e estruturas que moldam os fragmentos de mensagens (Bardin, 2016).

A pesquisa respeitou a Resolução n. 466 (2012), a qual postula que a ética em pesquisa deve respeitar a dignidade humana e a proteção a participantes envolvidos na pesquisa. Para tanto, o projeto foi submetido e aprovado em abril de 2020, 
pelo Comitê de Ética da Faculdades Pequeno Príncipe, em coparticipação do Comitê de Ética e Viabilidade da Secretaria Municipal de Saúde do Município de Curitiba, Paraná, Brasil, sob os Pareceres Consubstanciados n. 3.978.619 e n. 4.043.053.

\section{Resultados e Discussão}

Os achados deste estudo foram divididos em duas etapas de análise - PIs e Questionários. Nesta primeira etapa referente aos PIs revelou-se que as palavras-chaves pesquisadas aparecem restritas a um contexto de delimitação de público atendido e com normativas operacionais, conforme Quadro 1:

Quadro 1 - Projetos institucionais:

\begin{tabular}{|c|c|}
\hline Palavras-Chave & Núcleos de Significação \\
\hline $\begin{array}{l}\text { gênero(s), masculino, } \\
\text { feminino, homem, } \\
\text { mulher, transgênero, } \\
\text { expressão de gênero, } \\
\text { transexual, } \\
\text { homossexual, lésbica, } \\
\text { gay, bissexuais, } \\
\text { pansexual, LGBT+, } \\
\text { acolhimento, ofertas } \\
\text { terapêuticas. }\end{array}$ & $\begin{array}{l}\text { O acolhimento é realizado pela equipe multiprofissional escalada para o dia, sendo } \\
\text { disponibilizados por semana } 20 \text { atendimentos eletivos e } 20 \text { atendimentos emergenciais. } \\
\text { Estes em geral são agendados pelo telefone ou presencialmente (PI CAPS 03, p. 6). } \\
\text { As Ofertas Terapêuticas no CAPS [pesquisado] contemplam um conjunto de intervenções } \\
\text { realizadas pela equipe multiprofissional, que podem acontecer de forma individualizada ou } \\
\text { coletiva, de acordo com o Projeto Terapêutico Singular estabelecido (PI CAPS 02, p. 17). } \\
\text { A maioria dos usuários é do sexo masculino (PI CAPS 1, p. 6). } \\
\text { Há um aumento considerável da inserção de mulheres (PI CAPS 1, p. 7). } \\
\text { Quanto ao perfil dos usuários ativos são na maioria de homens, } 70 \% \text { (PI CAPS 1, p. 7). } \\
\text { Em relação ao gênero, 50,5\% da população regional é composta por mulheres ( PI CAPS } \\
\text { 2, p. 5). }\end{array}$ \\
\hline
\end{tabular}

Fonte: Dados da pesquisa (2020).

Identificou-se, apenas em um PI, a palavra "feminino" referindo-se a um grupo terapêutico - oferta terapêutica específica para mulheres:

Promover espaço de escuta grupal, possibilitando a expressão de sentimentos e emoções acerca do sofrimento psíquico, com foco nas questões voltadas ao feminino (CAPS 1, p. 23).

As palavras-chave: Gênero(s), Transgênero (s), Expressão de Gênero, Transexual, Lésbica, Gay, Assexual, Pansexual, LGBT+ (e a variação da sigla como LGBTQI) não foram localizadas em nenhum dos PIs analisados.

O PI de um CAPS se constitui como documento norteador, que embasa diretrizes, objetivos e ofertas terapêuticas dos dispositivos. Surge como uma estratégia social e política, que deve considerar o território e a cultura local (Ferreira \& Carvalho, 2017). Os PIs contemplam os principais saberes e tarefas e levam em conta as legislações vigentes da política de saúde mental (Vasconcelos et al., 2016). Dentre os marcos norteadores a Lei n. 10.216 (2001, Art.1) destaca que os direitos dos/as usuários/as devem ser assegurados independente de "raça, cor, sexo, orientação sexual, religião, opção política, nacionalidade, idade, família, recursos econômicos e ao grau de gravidade ou tempo de evolução de seu transtorno, ou qualquer outra". Assim também o Art. 2 da Portaria n. 3088 (2011) garante o respeito aos Direitos Humanos, autonomia e liberdade, promovendo a equidade e combatendo estigmas e preconceitos sociais. 
Tomando esses pressupostos para análise dos PIs, evidenciou que os mesmos norteiam o funcionamento e organização dos CAPS investigados, descrevem o uso de tecnologias leves de cuidado tais como acolhimento, escuta ativa e ambiência, caracterizam as ofertas terapêuticas, bem como, incluem orientações de manejo a crises e a construção dos Projetos Terapêuticos Singulares (Merhy, Magalhães Júnior, Rimoli, Franco, \& Bueno 2004). Contudo, na busca pelas palavras-chave, não houve identificação de qualquer abordagem relativa as diferentes expressões de gênero, a diversidade sexual (Ferreira, Coelho \& Duarte, 2018), ao patriarcado (Narvaz \& Koller, 2006), a heteronormatividade ou a questões de violência, fato que indica um distanciamento institucional destas questões emergentes na sociedade atual. Ao não ocupar-se deste tema nos documentos norteadores pressupõem-se a ausência de planejamento de intervenções no território adscrito, limitando a atuação dos serviços a ações "intra muros" e a uma perspectiva passiva de acolhimento.

Outro achado na analise dos PIs trata da oferta terapêutica identificada no CAPS 01, grupo terapêutico direcionado a "mulheres". Este grupo proposto fundamenta-se no referencial teórico psicanalítico, o qual pressupõe uma concepção fundada no binarismo homem/mulher (Butler, 2018), distante de discussões de inclusão das diferentes expressões de gênero em dispositivos voltados ao cuidado à Saúde Mental (Zanello, 2018). O que desperta o questionamento acerca de quais pressupostos epistemológicos tem fundado a atuação clínica, em especial de profissionais da Saúde Mental, tendo em vista que a atenção psicossocial pressupõe uma apropriação de contexto, historicidade, diversidade e ênfase na reinserção social de seus usuários/as (Almeida, 2019; Yasui \& Barzaghi, 2018). O aporte teórico identificado indica a ausência de uma discussão entre a clínica tradicional e a clínica emergente, aquela que se ocupa com aspectos sociais e atuais, torna-se necessário atendar-se que "acolher significa, acima de tudo, considerar as subjetividades como constituindo-se num mundo em que as dimensões históricas, sociais e culturais exercem o seu papel no processo de subjetivação. Esta seria a ética de uma nova postura clínica" (Dutra, 2004, p. 385).

\section{Segunda Etapa - Questionários}

Na segunda etapa foram aplicados 15 questionários, um excluído por preenchimento incompleto, totalizando 14 para análise. Tendo sido aplicado cinco questionários em cada CAPS. Quanto ao perfil de participantes, responderam à pesquisa: dois médicos/as, três enfermeiros/as, quatro psicólogos/as, dois terapeutas ocupacionais e três assistentes sociais e um não informado. Sendo excluído o questionário que não contém a informação. A média de idade foi de 29,5 anos, sendo obtida entre a menor, 26 anos, e a maior, 62. Referente à escolaridade, além da graduação, oito são pós-graduados/as. O tempo médio de atuação em CAPS foi de cinco anos. Dos respondentes $94 \%$ se declaram mulheres cis e $6 \%$ homens cis. Após aplicação da Análise de Conteúdo, chegou-se a três categorias, conforme Quadros 2,3 e 4: 
Quadro 2 - Categoria 1: Expressões de gênero e saúde mental.

\begin{tabular}{|c|c|}
\hline $\begin{array}{c}\text { Categorias } \\
\text { de } \\
\text { discussão }\end{array}$ & Núcleos de Significação \\
\hline $\begin{array}{c}\text { Expressões } \\
\text { Gênero e } \\
\text { Saúde } \\
\text { Mental }\end{array}$ & $\begin{array}{l}\text { As questões de gênero são importantes e contribuem significativamente para o adoecimento psíquico de } \\
\text { um número significativo de usuários (P4). } \\
\text { Sim, vemos, com frequência, pessoas que tem dificuldades em se expressar ou até mesmo em assumir sua } \\
\text { identidade e por isso apresentam sofrimento psíquico (P5). } \\
\text { Acredito que o adoecimento psíquico está diretamente associado a construção social do papel social do } \\
\text { gênero. O sofrimento psíquico está diretamente associado com todas as formas de violência e violação de } \\
\text { direitos que uma pessoa sofre ao não se encaixar no binarismo masculino e feminino (P8). } \\
\text { Questões de gênero têm impacto significativo desde o crescimento/desenvolvimento do usuário/usuária no } \\
\text { âmbito familiar. É muito comum a não-aceitação dessa expressão no âmbito familiar e demais ambientes } \\
\text { sociais. É comum também a não-validação de sentimentos/pensamentos do usuário. Para além de como a } \\
\text { sociedade e de como o outro recebe, há o sofrimento intrínseco a essa condição - por exemplo, não se } \\
\text { identificar com o próprio corpo, ter vontade de morrer por esse motivo e etc. (P3). } \\
\text { Sim. Muitas das vezes o ambiente que esta pessoa está inserida não é acolhedora e pode oferecer riscos } \\
\text { caso essa pessoa se expresse da forma desejada. Pode ocorrer do usuário não se aceitar e reprimir seus } \\
\text { desejos e provocar angustia ou negação. Já houve situaçães do usuário ter ideação suicida por não aceitar } \\
\text { sua própria expressão de gênero por já ter sofrido bullying até mesmo de profissionais no serviço (P14). } \\
\text { A equipe percebeu que as pessoas trans ou com expressões de gênero diferentes da normativa sofriam } \\
\text { preconceito na forma de piadas ou comentários maldosos, tanto pelos demais pacientes, quanto por alguns } \\
\text { funcionários (P12). } \\
\text { Já houve comentários de senso comum sobre determinados pacientes que precisaram ser discutidos e } \\
\text { relembrar a funcionários específicos que esse tipo de atitude não se encaixa com o serviço de saúde como o } \\
\text { CAPS (P14). }\end{array}$ \\
\hline
\end{tabular}

Fonte: Dados da pesquisa (2020).

Quando correlacionados os achados desta pesquisa a questões de Saúde Mental, o que se identificou foi a percepção dos/as profissionais, quanto às vulnerabilidades que envolvem a população LGBTQIA+. Associam estas vulnerabilidades a questões de autoaceitação, a relação familiar e ao contexto social. Nas respostas os participantes também sinalizam a violação de direitos e as diferentes formas de violências a que estão sujeitos este público, indicando todos estes fatores, como riscos ao adoecimento psíquico. Essa percepção é corroborada por diferentes estudos (Wolff \& Saldanha, 2015; Paveltchuk \& Borsa, 2019) e de acordo com Silvia Lane (2017) esta vulnerabilidade pode estar associada ao fato de que, grupos que não correspondem ao esperado socialmente estão mais sujeitos a discriminação, preconceito e segregação.

Na sociedade brasileira a diversidade sexual encontra-se marcada, tanto pelo pensamento judaico-cristão, que incute uma perspectiva de pecado à população LGBTQIA+, quanto uma forte influência do paradigma biomédico que herda dos manuais diagnósticos o conceito de normal e patológico, categorizando a diversidade como desajustamento (Zanello, 2014; Butler, 2018; Ferreira, Coelho \& Duarte, 2018). A sociedade contemporânea também reproduz o modelo patriarcal, que coloca a mulher num papel hierárquico de menor valor e muitas vezes subjugada ao homem (Zanello, 2014; Butler, 2018).

Esta discussão alerta ao fato de que aspectos sociais são determinantes para compreensão do processo saúde-doença e em especial, ao objeto deste estudo, ao adoecimento psíquico. A população LGBTQIA+ tem apresentado, segundo dados epidemiológicos, uma alta prevalência de quadros de ansiedade, depressão e comportamento suicida (Peres \& Toledo, 2011; Portaria n. 2836, 2011; Wolff \& Saldanha, 2015; Paveltchuk \& Borsa, 2019) que alertam profissionais da Saúde Mental ao compromisso ético-político de inclusão desta pauta na sua agenda e de uma atuação ativa na busca de oferecer cuidado. 
Contudo, profissionais da Saúde também estão sujeitos a reproduzir comportamentos discriminatórios, considerando sua visão de mundo, crenças e representações sociais compartilhadas por seus grupos (Zanello, 2018). Este fato foi deflagrado na coleta de dados e é corroborado por Zanello \& Silva (2012, p. 269) ao afirmar que "os olhares também são marcados pelos valores de gênero, os quais se tornam fatores determinantes na atividade hermenêutica da leitura dos sintomas". Estas ocorrências podem impactar em diferentes aspectos, desde a aderência a tratamentos de saúde mental, novas buscas a dispositivos de Saúde e mesmo a um agravamento do sofrimento psíquico (Paveltchuk \& Borsa, 2019).

Quadro 3 - Categoria 2: Gênero acolhimento e ofertas terapêuticas em CAPS.

\begin{tabular}{|c|c|}
\hline $\begin{array}{l}\text { Categorias de } \\
\text { discussão }\end{array}$ & Núcleos de Significação \\
\hline $\begin{array}{c}\text { Expressões de } \\
\text { Gênero Acolhimento } \\
\text { e Ofertas } \\
\text { Terapêuticas }\end{array}$ & $\begin{array}{l}\text { Não existe diferença na adesão. A procura pela população trans é pequena (P13). } \\
\text { Os casos são discutidos individualmente conforme a demanda de cada um (P1). } \\
\text { Sim, já foi discutido sobre usuários em específico e discutido acerca do plano terapêutico dele. Sempre } \\
\text { respeitando como a pessoa se identifica (P5). } \\
\text { Sim, quando esta temática tem relação direta com as queixas e demandas apresentadas pelo indivíduo } \\
\text { (P6). } \\
\text { Uma das ferramentas fundamentais utilizadas para a inserção do usuário no serviço do CAPS e adesão } \\
\text { ao tratamento proposto é o ACOLHIMENTO, conceito que se refere a uma ação voltada a } \\
\text { aproximação, uma atitude que tem como objetivo a inclusão do paciente a uma oferta de serviço da } \\
\text { saúde (p. 13). } \\
\text { O atendimento é realizado como para todos, acredito que não são diferenciados em recepção e inserção } \\
\text { no serviço (P2). } \\
\text { O acolhimento, atendimento e escolha de ofertas terapêuticas acontece como o é com qualquer usuário } \\
\text { do serviço (P3). } \\
\text { As ofertas terapêuticas são direcionadas ao 'ser humano' onde se procura respeitar o paciente de acordo } \\
\text { com suas características individuais (P15). } \\
\text { O acolhimento é realizado da mesma forma que os outros usuários. As ofertas terapêuticas são } \\
\text { definidas por projetos terapêuticos singular, de forma individualizada. Caso a expressão de gênero seja } \\
\text { algo que o paciente considere importante no seu tratamento isso será abordado em seus atendimentos. } \\
\text { Cabe lembrar que é o usuário que traz a demanda de atendimento, de forma que isso não será abordado } \\
\text { se não for do seu interesse (P4). } \\
\text { a maneira como o serviço funciona deveria ser neutra, independente da identidade de gênero, porém, } \\
\text { muitas vezes presencio profissionais impondo seu discurso pessoal e/ou religioso para os usuários, de } \\
\text { forma que deixa o paciente extremamente desconfortável (P14). }\end{array}$ \\
\hline
\end{tabular}

Fonte: Dados da pesquisa (2020).

$\mathrm{O}$ acolhimento e as ofertas terapêuticas são tecnologias leves de cuidado, ditas tecnologias relacionais. O acolhimento é utilizado no contato com usuários/as, proporciona uma escuta humanizada de suas demandas, visa embasar a análise do caso e a escolha das ofertas terapêuticas (Oliveira et al., 2019; Jorge et al., 2011; Romanini, Guareschi \& Roso, 2017). O momento do acolhimento pode afetar a permanência do/a usuário/a no tratamento e a sua adesão ao Serviço de Saúde (Oliveira et al., 2019; Jorge et al., 2011; Romanini, Guareschi \& Roso, 2017).

As ofertas terapêuticas são proporcionadas após o acolhimento das principais demandas do sujeito, embasadas de forma que proponham o compartilhamento das experiências individuais no meio coletivo, sem rotular o sujeito pelo sofrimento psíquico que apresenta. Desta forma, ao escolher as ofertas terapêuticas, profissionais devem levar em conta a vivência do 
sujeito e o meio social no qual está inserido (Dias, 2018), proporcionando formas de convivência e acompanhamento longitudinal (Ibiapina et al., 2017).

Os achados deste estudo indicam um ajustamento das práticas dos/as profissionais dos CAPS a estes pressupostos, contudo, quando se investiga especificamente questões relacionadas a diferentes expressões de gênero, esse tema não toma destaque nas respostas, sendo apontado como relevante quando explicitado pelo/a usuário/a. Não foi identificada em nenhuma resposta uma postura mais ativa de investigação referente a questões de gênero no acolhimento, bem como a sua observância na escolha de ofertas terapêuticas.

Também, destaca-se, não houve nesta pesquisa o reconhecimento de estratégias dos CAPS que busquem identificar essa demanda no território. Tampouco, foram identificadas estratégias busquem facilitar o acesso destas pessoas aos serviços, embora seja reconhecida pelos/as profissionais a vulnerabilidade da população LGBTQIA+ e estudos apontem para os riscos de adoecimento psíquico.

\section{Considerações Finais}

Este estudo compreendeu que as diferentes expressões de gênero não são tomadas a priori como aspecto relevante, no acolhimento e na escolha de ofertas terapêuticas, por profissionais de saúde que atuam nos CAPS pesquisados. Tal incorporação ocorre somente quando esta demanda se apresenta de forma explícita pelo/a usuário/a e mais, quando este/a chega ao acolhimento no CAPS. Embora os/as profissionais identifiquem a vulnerabilidade relacionada à vivência de diferentes expressões de gênero, não foi possível identificar estratégias institucionais voltadas ao tema.

Esta pesquisa beneficia a problematização acerca da natureza dos CAPS enquanto agentes promotores da Saúde Mental em seu território, extrapolando uma ação centrada na doença e nos/as usuários/as atendidos/as por demandas de transtornos mentais graves e/ou crônicos, convocando profissionais a protagonizar ações e articulações que beneficiem o acesso e a adesão ao cuidado a diferentes ofertas de Saúde Mental e assim, resgatar os pressupostos psicossociais de garantia de direitos e reinserção social.

O estudo apresentou limitações por ter sido realizado no período de pandemia da Doença do Coronavírus - 19 (COVID-19), o que inviabilizou a realização de entrevistas que permitiriam um aprofundamento da questão norteadora; foi necessário optar pela aplicação de questionários, respeitando os protocolos sanitários de segurança. Sugere-se reproduzir este estudo em outros CAPS, de outros territórios, ampliando o conhecimento de como profissionais da Saúde Mental tem abordado as questões de gênero. Esta pesquisa também despertou o interesse em buscar narrativas de usuários/as do Sistema Único de Saúde para cotejar percepções e apropriações, tendo em vista, a responsabilidade social dos/as profissionais de saúde de proporcionar acesso e efetividade nas suas ações.

\section{Agradecimentos}

Agradecemos o apoio das Faculdades Pequeno Príncipe e ao acolhimento dos Centros de Atenção Psicossocial envolvidos na pesquisa.

\section{Referências}

Amarante, P. (2015). Saúde mental e atenção psicossocial. 4. ed. Rio de Janeiro: Fiocruz.

Amarante, P. \& Nunes, M. O. (2018). A reforma psiquiátrica no SUS e a luta por uma sociedade sem manicômios. Revista Ciênc. saúde coletiva, 23(6), 20672074.

Andrade, A. P. M. (2014). (Entre)Lançamentos possíveis entre gênero e saúde mental. In V. Zanello, \& A. P. M. Andrade (Orgs.). Saúde mental e gênero: diálogo, práticas e interdisciplinaridade (pp. 59-77). Curitiba: Appris. 
Andrade, L. H. S. G., Viana, M. C., \& Silveira, C. M. (2006). Epidemiologia dos transtornos psiquiátricos na mulher. Rev. Psiq. Clín., 33(2), 43-54.

Lei n. 10.216, de 6 de abril de 2001 (2001). Dispõe sobre a proteção e os direitos das pessoas portadoras de transtornos mentais e redireciona o modelo assistencial em saúde mental. Recuperado em 04 agosto, 2020, de http://www.planalto.gov.br/ccivil_03/leis/leis_2001/110216.htm.

Almeida, J.M.C. (2019). Políticas de saúde mental no Brasil: o que está em jogo nas mudanças em curso. Cad. Saúde Pública, 35(11):e00129519. https://www.scielo.br/j/csp/a/KMwv8DrW37NzpmvL4WkHcdC/?lang=pt\&format=pdf.

Portaria n. 2836, de $1^{\circ}$ de dezembro de 2011 (2011). Institui, no âmbito do Sistema Único de Saúde (SUS), a Política Nacional de Saúde Integral de Lésbicas, Gays, Bissexuais, Travestis e Transexuais (Política Nacional de Saúde https://bvsms.saude.gov.br/bvs/saudelegis/gm/2011/prt2836_01_12_2011.html.

Portaria n. 3088, de 23 de dezembro de 2011 (2011). Recuperado em 04 agosto, 2020, de http://bvsms.saude.gov.br.

Resolução n. 466 de 12 de dezembro de 2012. Recuperado em 04 agosto, 2020, de https://conselho.saude.gov.br/resolucoes/2012/Reso466.pdf.

Butler, J. (2018). Problemas de gênero: feminismo e subversão da identidade. 16. ed. Rio de Janeiro: Civilização Brasileira.

Bardin, L (2016). Análise de Conteúdo. São Paulo: Edição 70.

Decreto n. 638 de junho de 2018 (2018). Institui o Índice de Vulnerabilidade das Áreas de Abrangência das Unidades Municipais de Saúde - IVAB na Secretaria Municipal da Saúde de Curitiba Diário Oficial Eletrônico - Atos do Munícipio de Curitiba, jun. 2018 . https://diarios.s3.amazonaws.com/DOMCTBA/2018/06/Normal/pdf/20180621_42.pdf?AWSAccessKeyId=AKIAI45WSOUE2QEAQ6HQ\&Expires=1557099 365\&Signature=TiL51sgkKWGsDVBH\%2B2MELhWhWyQ\%3D.

Dutra, E. Considerações sobre as significações da psicologia clínica na contemporaneidade. Dossiê: Práticas psicológicas em instituições: atenção, desconstrução e invenção. Estudos psicol.. Natal 9(2). https://www.scielo.br/j/epsic/a/7dTyvpTbPQW9XfFsgk4shen/?lang=pt.

Ferreira, L. C. M., Coelho, M. O., \& Duarte, M. J. (2018). Diversidade sexual e de gênero na saúde mental: aproximações e experiências no campo da pesquisa. REBEH, 2(1), 2018. www.revistas.unilab.edu.br/index.php/rebeh.

Gil, A. C. (2017). Como elaborar projetos de pesquisa. 6. ed. São Paulo: Atlas.

Ibiapina, A. R. S., Monteiro, C. F. S., Alencar, D. C., Fernandes, M. A., \& Costa Filho, A. A. I. (2017). Oficinas terapêuticas e as mudanças sociais em pacientes com transtornos mentais. Esc. Anna Nery, 21(3). DOI: https://doi.org/10.1590/2177-9465-EAN-2016-0375

Jorge, M. S. B., Pinto, D. M., Quinderé, P. H. D., Pinto, A. G. A., Sousa, F. S. P., \& Cavalcante, C. M. (2011). Promoção da saúde mental - tecnologias do cuidado: vínculo, acolhimento, co-responsabilização e autonomia. Ciênc. saúde coletiva, 16(7). DOI: https://doi.org/10.1590/S1413-81232011000800005.

Lane, S. T. M. (2017). O que é psicologia social. São Paulo: Brasiliense.

Lima, R. L. (2011). Diversidade, identidade de gênero e religião: algumas reflexões. EM PAUTA, 9(2), 165-182. https://www.epublicacoes.uerj.br/index.php/revistaempauta/article/view/2940/2104.

Maas, S. O., \& Savaris, L. E. (2020). Centros de Atenção Psicossocial e expressões de gênero. Anais VI Simpósio Gênero e Políticas Públicas. http://anais.uel.br/portal/index.php/SGPP/article/view/1084/1053.

Marconi, M. A., \& Lakaos, E. M. (2021). Fundamentos de metodologia científica. 9. ed. São Paulo: Atlas.

Merhy, E. E., Magalhães Júnior, H. M., Rimoli, J., Franco, T. B., \& Bueno, W. S. (2004). O trabalho em saúde: olhando e experenciando o SUS no cotidiano. 2. ed. São Paulo: Hucitec.

Moscovici, S. ([1961]2011). Psicologia das minorias ativas. Petrópolis: Vozes.

Moscovici, S. (2019). Representações sociais: investigação em psicologia social. 11. ed. Petrópolis:Vozes.

Narvaz, M. G., \& Koller, H. (2006). Famílias e patriarcado: da prescrição normativa à subversão criativa. https://www.scielo.br/j/psoc/a/VwnvSnb886frZVkPBDpL4Xn/abstract/?lang=pt.

Nascimento, W. F., Zanello, V. (2014). Uma história do silêncio sobre gênero e loucura - Parte I. Sobre o que não se fala em uma arqueologia do silêncio: as mulheres em História da Loucura (pp. 17-28). In V. Zanello, \& A. P. M. Andrade. (Orgs.). Saúde mental e gênero: diálogo, práticas e interdisciplinaridade. Curitiba: Appris.

Paveltchuk, F. O., \& Borsa, J. C. (2019). Homofobia internalizada, conectividade comunitária e saúde mental em uma amostra de indivíduos LGB brasileiros. Avances En Psicología Latinoamericana, 37(1), 47-61.

Peres, W. S., \& Toledo, L. G. (2011). Dissidências existenciais de gênero: resistências e enfrentamento ao biopoder. Rev. psicol. polít.,11(22), 261-277. http://pepsic.bvsalud.org/scielo.php?script=sci_arttext\&pid=S1519549X2011000200006\&lng=pt\&nrm=iso.

Rabasquinho, C.; Pereira, H (2007). Gênero e saúde mental: uma abordagem epidemiológica. Aná. Psicológica, Lisboa, v. 25, n. 3, p. 439-454. http://www.scielo.mec.pt/scielo.php?script=sci_arttext\&pid=S087082312007000300010\&lng=pt\&nrm=iso.

Dias, J. D. S. (2018) Oficinas Terapêuticas como Estratégia para Reinserção Psicossocial e Produção de Vínculo. Pretextos - Revista da Graduação em Psicologia da PUC Minas, v. 3, n. 5, p. 129-145. http://periodicos.pucminas.br/index.php/pretextos/article/view/15933. 
Research, Society and Development, v. 10, n. 13, e133101320836, 2021

(CC BY 4.0) | ISSN 2525-3409 | DOI: http://dx.doi.org/10.33448/rsd-v10i13.20836

Ferreira, K.F. \& Carvalho, V. C. S. (2017) Oficinas terapêuticas: caminhos de saberes. Revista da Faculdade de Ciências Medicas de Sorocava. file://C:/Users/Suelen\%20Maas/Desktop/Revista/32995-Texto\%20do\%20artigo-106939-1-10-20180719.pdf.

Romanini, M., Guareschi, P. A., \& Roso, A. (2017). O conceito de acolhimento em ato: reflexões a partir dos encontros com usuários e profissionais da rede. Saúde debate, 41(113), 486-499.

Santos, A. M. C. C. (2009). Articular saúde mental e relações de gênero: dar voz aos sujeitos silenciados. Ciênc. saúde coletiva, 14(4), $1177-1182$.

Secretaria de Justiça e da Defesa da Cidadania (2004). Diversidade sexual e a cidadania LGBT. Imprensa Oficial do Estado de São Paulo.

Oliveira, A.B., Barbosa, V.F.B., Alexandre A.C.S., Santos, D.C.S., Costa, L.S., Pereira, L.C.S., Leite, J.A. (2019). Acolhimento na Rede de Atenção Psicossocial: Revisão Integrativa de Literatura. Rev. Mult. Psic. V.13, n.45 $^{\text {Suplemento }} 1$, p.318-332. https://idonline.emnuvens.com.br/id/article/view/1844/2672.

Vasconcelos, M.G.F., Jorge, M.S.B., Catrib, A.M.F., Bezerra, I.C., \& Franco, T.B. (2016). Projeto terapêutico em Saúde Mental: práticas e processos nas $\begin{array}{llllll}\text { dimensões } & \text { constituintes } & \text { de } & \text { atenção } & \text { psicossocial. } & \text { Interface, }\end{array}$ https://www.scielo.br/j/icse/a/ngWyPTTHnnsj4hdVmxn8fqC/?format=pdf\&lang=pt.

Wolff, C. S., \& Saldanha, R. A. (2015). Gênero, sexo, sexualidade: categorias do debate contemporâneo. Revista Retratos da Escola, 9(16), 29-46.

Yassui, S., \& Barzaghi, N. (2018). História, Memória e Luta: A construção da Reforma Psiquiátrica no Brasil. Convención Internacional de Salud, Cuba Salud. http://convencionsalud2018.sld.cu/index.php/connvencionsalud/2018/paper/viewFile/792/895.

Zanello, V., \& Silva, R. M. C. (2012). Saúde mental, gênero e violência estrutural. Rev. bioét.,20(2), 267-279. https://revistabioetica.cfm.org.br/index.php/revista_bioetica/article/view/745.

Zanello, V. (2014). Saúde mental sob o viés do gênero: uma releitura engendrada da epidemiologia, da semiologia e da interpretação diagnóstica. (pp. 41-58). In V. Zanello, \& A. P. M. Andrade (Orgs.). Saúde mental e gênero: diálogo, práticas e interdisciplinaridade. Curitiba: Appris.

Zanello, V. (2018). Saúde mental, gênero e dispositivos: cultura e processos de subjetivação. Curitiba: Appris. 